\title{
Maternal Factors Associated with Family Social Distancing Practices During the Coronavirus Disease-2019 Pandemic
}

\author{
Christine A. Limbers ${ }^{1}$ (D) Emma Summers $^{1}$ \\ Accepted: 7 August 2021 / Published online: 17 August 2021 \\ (c) The Author(s), under exclusive licence to Springer Science+Business Media, LLC, part of Springer Nature 2021
}

\begin{abstract}
Objectives Despite social distancing being an effective method for mitigating community transmission of viruses, little is known about factors associated with social distancing practices among children and their families. The current study assessed maternal socio-demographic characteristics and political party identifications associated with family social distancing practices during the COVID-19 pandemic.

Methods Participants in this study were 1266 mothers (mean age $=39.92$ years; $84.9 \%$ white) of children ages 17 years and younger from across the United States. They were recruited online through social media platforms and completed questionnaires on Qualtrics about their family's social distancing practices and socio-demographic characteristics.

Results Women with a Doctorate (mean $=35.37 ; \mathrm{SD}=4.24$ ) and Master's (mean =34.26; $\mathrm{SD}=5.70$ ) degree reported higher levels of family social distancing compared to women with some college (mean $=31.11 ; \mathrm{SD}=8.11)$ or a college degree $($ mean $=32.62 ; \mathrm{SD}=6.91 ; p$ 's $=.00)$. Women who identified as Democrat (mean $=35.92 ; \mathrm{SD}=3.30)$ or Independent (mean 34.13; $\mathrm{SD}=5.63)$, or indicated not identifying with a political party (mean $=34.19 ; \mathrm{SD}=5.69)$, reported higher levels of family social distancing compared to women who identified as Republican (mean $=29.70 ; \mathrm{SD}=8.12 ; p$ 's $=.00$ ). The largest effect was found between women who identified as Democrat and Republican (effect size $=1.00$ ). After controlling for relevant predictor variables, maternal education (standardized beta coefficient $=.116 ; p=.000$ ), race (standardized beta coefficient $=.072 ; p=.007$ ), and political party identification (standardized beta coefficient $=-.348 ; p=.000$ ) were significantly correlated with the Social Distancing Total Score.

Conclusions for Practice The current findings suggest there may be a benefit to COVID-19 public health campaigns targeting families with lower educational attainment and more conservative regions in the United States.
\end{abstract}

Keywords COVID-19 $\cdot$ Social distancing $\cdot$ Maternal factors $\cdot$ Families $\cdot$ Children

\section{Significance}

Social distancing practices are important for mitigating transmission of COVID-19. Little is known about factors associated with social distancing practices among children and their families, who may be among the most mobile members of society and experience the greatest challenges with social distancing during the COVID-19 pandemic. The current study investigates maternal socio-demographic characteristics and political party identifications associated

Christine A. Limbers

Christine_Limbers@baylor.edu

1 Department of Psychology and Neuroscience, Baylor University, One Bear Place \#97334, Waco, TX 76798, USA with family social distancing practices during the COVID19 pandemic.

\section{Introduction}

The Coronavirus Disease-2019 (COVID-19) was declared a pandemic in the United States (U.S.) on March 19, 2020. Social distancing practices, which promote decreased infectious contact in communities, are an important strategy for mitigating transmission of COVID-19 (CDC, 2020; Courtemanche et al., 2020). In a recent systematic review and meta-analysis (Chu et al., 2020), physical distancing of $1 \mathrm{~m}$ or greater was found to be moderately effective in lowering transmission of SARS-CoV-2 and the betacoronaviruses that lead to severe acute respiratory syndrome. Mask wearing 
resulted in large reductions in risk of virus transmission, with $\mathrm{N} 95$ and other respirator devices providing greater protection than disposable surgical masks (Chu et al., 2020). Another systematic review that examined social distancing measures during the 2009 influenza pandemic (Rashid et al., 2015) found that closing schools, regardless if reactive or proactive, was moderately effective in slowing the spread of influenza; however, closing schools was associated with substantial secondary costs including loss of income for families whose parents had to take time off from work to care for their children and children not receiving adequate educational opportunities and services including free or reduced cost meals. Voluntary quarantine/home isolation was another effective social distancing strategy, although it was associated with an elevated risk for within household virus transmission (Rashid et al., 2015).

Despite social distancing being an effective method for mitigating community transmission of viruses, little is known about factors associated with social distancing practices among children and their families, who may be among the most mobile members of society and experience the greatest challenges with social distancing during the COVID-19 pandemic. There are data that indicate adults who identify politically as Conservative are less likely to practice physical distancing during the COVID-19 pandemic (Clinton et al., 2020; Gollwitzer et al., 2020; Leventhal et al., 2021). As such, elucidating political party identifications and socio-demographic characteristics associated with social distancing practices in children and families could aid in the development of public health campaigns that target members of society who are the least likely to abide by social distancing recommendations. Further, such information could assist schools and organizations that offer extra-curricular activities (e.g., organized sports, church youth groups) in developing safety plans that involve social distancing practices. The purpose of the current study was to assess maternal socio-demographic characteristics and political party identifications associated with family social distancing practices in a national sample of women.

\section{Methods}

\section{Participants}

Participants in this cross-sectional study were a convenience sample of 1266 women recruited online through social media platforms. To be eligible to participate in the study, women had to be from the U.S. and have at least one child 17 years old or younger living with them at least $50 \%$ of the time. Women who were not literate in English were excluded from the study.

Table 1 Items on the Social Distancing Questionnaire

Item

1. Before my family and I go out, we know and follow the guidance from local public health authorities where we live

2. When running errands or commuting to and from work, my family and I utilize social distancing options such as trying to keep at least 6 feet from other passengers or transit operators; when using rideshares or taxis, we avoid pooled rides where multiple passengers are picked up, and sit in the back seat in larger vehicles so we can remain at least 6 feet away from the driver

3. My family and I only visit stores selling household essentials in person when we absolutely need to, and stay at least 6 feet away from others who are not from our household while shopping and in lines

4. If possible, my family and I use drive-thru, curbside pick-up, or delivery services to limit face-to-face contact with others. We maintain physical distance between ourselves and delivery service providers during exchanges and wear a cloth face covering

5. My family and I stay socially connected with friends and family who don't live in our home by calling, using video chat, or staying connected through social media

6. If meeting others in person (e.g., at small outdoor gatherings, yard or driveway gathering with a small group of friends or family members), my family and I stay at least 6 feet from others who are not from our household

7. My family and I avoid crowded places and gatherings where it may be difficult to stay at least 6 feet away from others who are not from our household

8. If my family and I have to be in a crowded space, we try to keep 6 feet of space between ourselves and others at all times, and wear a cloth face coverings; we pay attention to any physical guides, such as tape markings on floors or signs on walls, directing attendees to remain at least 6 feet apart from each other in lines or at other times

9. When my family and I go for a walk, bike ride, or wheelchair roll in our neighborhood or in another safe location, we make sure we can maintain at least 6 feet of distance between ourselves and other pedestrians and cyclists

10. When deciding to visit a nearby park, trail, or recreational facility, my family and I consider how many other people might be there and choose a location where it will be possible to keep at least 6 feet of space between ourselves and other people who are not from our household

Items on the Social Distancing Questionnaire were adapted from the Centers for Disease Control and Prevention (CDC) COVID-19 Tips for Social Distancing website (https://www.cdc.gov/coronavirus/2019-ncov/prevent-getting-sick/social-distancing.html). Response options were never (0), almost never (1), sometimes (2), almost always (3), always (4) 


\section{Measures}

\section{Social Distancing}

Participants completed a 10-item social distancing questionnaire that was adapted from the Centers for Disease Control and Prevention (CDC) COVID-19 Tips for Social Distancing Website (CDC, 2020). Table 1 presents the items on this questionnaire. Responses were scored on a 5-point Likert scale ranging from 0 (Never) to 4 (Always). Items were summed together to produce a Total Social Distancing Score, which represents the most comprehensive and reliable indicator of social distancing. Higher scores on the Total Social Distancing Score were indicative of greater family adherence to CDC recommended social distancing practices. Cronbach's Alpha for the Total Social Distancing Score in the current study was .91.

\section{Socio-demographic Information}

Participants completed a socio-demographic questionnaire that assessed the following maternal information: age, number of children 17 years and younger, ages of children, race (white, Black, Hispanic, Asian, Other), overall household annual income (less than $\$ 10,000, \$ 10,000-\$ 19,999$, $\$ 20,000-\$ 29,999, \$ 30,000-\$ 39,999, \$ 40,000-\$ 49,999$, $\$ 50,000-\$ 74,999, \$ 75,000-\$ 99,999, \$ 100,000-\$ 150,000$, More than $\$ 150,000$ ), marital status (married, divorced, separated, widowed, other), highest level of education (high school degree, some college, college degree, master's degree, doctoral degree), state residing in, and employment status. Participants were also asked if any of their children had a chronic health condition and whether anyone in their home had been diagnosed with COVID-19.

\section{Political Party Identification}

Participants were asked one question about which political party they identify with. Response options were Democrat, Republican, Independent, Other, and I do not identify with a political party.

\section{Procedures}

Participants were recruited between July 11 and August 11, 2020 through the social media platforms Facebook and Twitter. An advertisement was posted on a number of Facebook group pages relevant to women that read, "Are you a mother of at least one child 17 years old or younger? Researchers at $\mathrm{X}$ University want to learn more about maternal perceptions on social distancing. If you are interested in participating in this brief, 10-min online survey, please click on the link below." This study advertisement was also shared on the $\mathrm{X}$
University Science of Virtues Twitter account. Potential participants who clicked on the link were presented with a short description of the study and consent form. If they wanted to continue with the study, they were asked to provide their consent to participate through checking a box on the screen. The first three questions administered to participants were screening questions to confirm participants met the study inclusion criteria. Only participants who selected they were female and had at least one child 17 years old or younger that lived with them at least $50 \%$ of the time were permitted to proceed with the full study measures. Questionnaires were then completed by participants online through Qualtrics. One item was administered half-way through the survey as a validity check. This item read, "If you are reading this question, please select response option C below." Participants did not receive any financial compensation for their participation in the study. These study procedures were approved by the Institutional Review Board at X University were performed in accordance with the ethical standards laid down in the 1964 Declaration of Helsinki and its later amendments. All participants provided their informed consent prior to their inclusion in the study and any details that might disclose the identity of the participants were omitted.

\section{Statistical Analysis}

Descriptive statistics, including means and standard deviations, were computed for the social distancing questionnaire. Independent samples $t$-tests were computed to assess if there were statistically significant mean differences on the Social Distancing Total Score based on marital status (married vs. not married), race (white vs. non-white), child chronic health condition status (having a child with a chronic health condition vs. not having a child with a chronic health condition), whether anyone in the home had been diagnosed with COVID-19 (yes vs. no), and maternal employment status (employed outside the home vs. not employed outside the home). One-Way Analysis of Variance (ANOVA) with Tukey Post-Hoc Tests were computed to asses if there were statistically significant mean differences on the Social Distancing Total Score based on maternal education, combined annual income, political affiliation, and maternal age groups (18-29, 30-39, 40+ years). When there was a statistically significant mean difference, effect sizes were computed to determine the magnitude of the difference. Effect sizes for differences in means were designated as small (.20), medium (.50), and large (.80) in magnitude. Multiple linear regression analysis was used to examine multivariate associations between family social distancing practices, maternal sociodemographic characteristics, and political party identification. The Social Distancing Total Score was the criterion variable in the regression model. Maternal education, combined annual income, political affiliation, maternal race, and 
maternal age were entered into the regression analysis as predictor variables. All statistical analyses were conducted using SPSS Version 26 and a $p$ value of less than .05 was considered statistically significant.

\section{Results}

\section{Participant Characteristics}

A total of 1377 individuals consented to participation in the study. Of these individuals, 13 were not eligible to participate in the study because they reported their gender as male, 24 were not eligible because they did not have at least one child 17 years or younger, and 50 were not eligible to participate because their child or children did not live with them at least $50 \%$ of the time. Another 23 individuals did not respond to at least one of the required screening questions and as a result were not permitted to continue with the full set of questionnaires. Three individuals did not respond as instructed to the validity check item. Thus, the final sample was comprised of 1266 women.

Table 2 contains the socio-demographic characteristics of the sample. The mean age of women in the sample was 39.92 years $(\mathrm{SD}=5.99$; Range $=25-68$ years $)$. Number of children ranged from 1 to 7 children with a mean of 2 children $(\mathrm{SD}=.92)$. In terms of race, $84.9 \%$ of women identified as white, $5.4 \%$ as Hispanic, $3.8 \%$ as Asian, $1.1 \%$ as Black, and $1.6 \%$ as Other. The majority of women reported living in Texas (58.4\%), but all other states were represented (see Table 3). With regard to educational attainment, $1 \%$ of the women had earned a high school degree, $6.9 \%$ had attended some college, $34.4 \%$ held a college degree, $31.3 \%$ held a master's degree, and $23.3 \%$ held a doctoral degree. In the sample, $89.5 \%$ of the women reported being married, $4.3 \%$ divorced, $1.2 \%$ separated, and $3 \%$ widowed. In the sample, $12.2 \%$ of the women reported having a child with a chronic health condition, asthma being the most prevalent condition (7.4\%). Most women identified as a Democrat in terms of political party affiliation (38.5\%); $24.3 \%$ identified as Republican, $9.6 \%$ as Independent, and $22 \%$ did not identify with a political party. In terms of combined annual income, the majority of women $(83.5 \%)$ reported an annual income of at least $\$ 75,000$ per year. With regard to employment status, $72.1 \%$ of women reported working outside the home, of which $60.7 \%$ reported working full-time.

\section{Descriptive Statistics}

Overall, the percentage of missing data on the Social Distancing Questionnaire was 9.2\%. Little's test was used to evaluate missing data and data that were missing at random were imputed using expectation-maximization (EM). The
Table 2 Demographic variables for the sample

\begin{tabular}{|c|c|c|c|}
\hline Characteristic & $N$ or mean & $\%$ or SD & Range \\
\hline Number of children & 2.05 & .919 & $1-7$ \\
\hline 1 & 352 & 27.8 & - \\
\hline 2 & 594 & 46.9 & - \\
\hline 3 & 238 & 18.8 & - \\
\hline 4 & 58 & 4.6 & - \\
\hline 5 & 12 & 0.9 & - \\
\hline 6 & 4 & 0.3 & - \\
\hline 7 & 3 & 0.2 & - \\
\hline Missing & 5 & 0.4 & - \\
\hline \multicolumn{4}{|c|}{ Maternal marital status } \\
\hline Married & 1133 & 89.5 & - \\
\hline Divorced & 54 & 4.3 & - \\
\hline Separated & 15 & 1.2 & - \\
\hline Widowed & 4 & 0.3 & - \\
\hline Other & 19 & 1.5 & - \\
\hline \multicolumn{4}{|l|}{ Maternal education } \\
\hline High school degree & 13 & 1 & - \\
\hline Some college & 87 & 6.9 & - \\
\hline College degree & 435 & 34.4 & - \\
\hline Master's degree & 396 & 31.3 & - \\
\hline Doctoral degree & 295 & 23.3 & - \\
\hline Missing & 40 & 3.2 & \\
\hline \multicolumn{4}{|l|}{ Race } \\
\hline White & 1075 & 84.9 & - \\
\hline Black & 14 & 1.1 & - \\
\hline Asian & 48 & 3.8 & - \\
\hline Hispanic & 68 & 5.4 & - \\
\hline Other & 20 & 1.6 & - \\
\hline Missing & 41 & 3.2 & - \\
\hline Maternal age & 39.92 & 5.99 & $25-68$ \\
\hline \multicolumn{4}{|c|}{ Child with a chronic health condition } \\
\hline Yes & 155 & 12.2 & - \\
\hline No & 1067 & 84.3 & - \\
\hline Missing & 44 & 3.5 & - \\
\hline \multicolumn{4}{|c|}{ Anyone in home that had Covid19 } \\
\hline Yes & 33 & 2.6 & - \\
\hline No & 1191 & 94.1 & - \\
\hline Missing & 42 & 3.3 & - \\
\hline \multicolumn{4}{|c|}{$\begin{array}{l}\text { Someone with Covid } 19 \text { in the home } \\
\text { required hospitalization }\end{array}$} \\
\hline Yes & 3 & & - \\
\hline No & 30 & & - \\
\hline \multicolumn{4}{|c|}{ Employed outside the home } \\
\hline Yes & 913 & 72.1 & - \\
\hline No & 308 & 24.3 & - \\
\hline Missing & 45 & 3.6 & - \\
\hline \multicolumn{4}{|c|}{ Full or part time employment } \\
\hline Full time & 769 & 60.7 & - \\
\hline Part time & 142 & 11.2 & - \\
\hline Combined annual inc & & & \\
\hline
\end{tabular}


Table 2 (continued)

\begin{tabular}{llll}
\hline Characteristic & $N$ or mean & $\%$ or SD & Range \\
\hline Less than $\$ 10,000$ & 1 & 0.1 & - \\
$\$ 10,000-\$ 19,999$ & 5 & 0.4 & - \\
$\$ 20,000-\$ 29,999$ & 6 & 0.5 & - \\
$\$ 30,000-\$ 39,999$ & 18 & 1.4 & - \\
$\$ 40,000-\$ 49,999$ & 21 & 1.7 & - \\
$\$ 50,000-\$ 74,999$ & 100 & 7.9 & - \\
$\$ 75,000-\$ 99,000$ & 164 & 13 & - \\
$\$ 100,000-\$ 150,000$ & 418 & 33 & - \\
More than $\$ 150,000$ & 475 & 37.5 & - \\
Missing & 58 & 4.6 & - \\
Political party & & & \\
Democrat & 488 & 38.5 & - \\
Republican & 308 & 24.3 & - \\
Independent & 122 & 9.6 & - \\
Other & 23 & 1.8 & - \\
Did not identify with a political party & 279 & 22 & - \\
Missing & 46 & 3.6 & - \\
\hline
\end{tabular}

overall mean for the Social Distancing Questionnaire was $33.71(\mathrm{SD}=6.08)$.

\section{Independent Samples T-Test}

Compared to white women $(\mathrm{n}=1075)$, non-white women $(n=150)$ reported higher levels of social distancing for their families (white mean $=33.44 ; \mathrm{SD}=6.39$; non-white mean $=35.72 ; \mathrm{SD}=3.79 ; \mathrm{p}=.00$; effect size $=.43$ ). Compared to women who reported someone in their home had been diagnosed with COVID-19 $(n=33)$, women who reported no one in their home had been diagnosed with COVID-19 $(n=1191)$ reported higher levels of social distancing for their families (COVID-19 mean $=30.90$; $\mathrm{SD}=7.37$; non-COVID-19 mean $=33.79 ; \mathrm{SD}=6.13$; $\mathrm{p}=.01$; effect size $=.43$ ).

Married $(n=1133)$ and non-married $(n=92)$ women reported similar levels of social distancing for their families (married mean $=33.72 ; \mathrm{SD}=6.20$; non-married mean $=33.71 ; \mathrm{SD}=5.94 ; \mathrm{p}=.99)$. Mothers of at least one child with a chronic health condition $(n=155)$ and mothers without a child with a chronic health condition $(n=1067)$ reported similar levels of social distancing for their families (chronic health condition mean $=34.57 ; \mathrm{SD}=6.00$; non-chronic health condition mean $=33.59 ; \mathrm{SD}=6.20$; $\mathrm{p}=.06)$. Women who were employed $(\mathrm{n}=913)$ and women who were not employed $(n=308)$ reported similar levels of social distancing for their families (employed mean $=33.87$; $\mathrm{SD}=5.94$; non-employed mean $=33.21 ; \mathrm{SD}=6.84 ; \mathrm{p}=.11$ ).
Table 3 States participants resided in

\begin{tabular}{|c|c|c|}
\hline State & $N$ & $\%$ \\
\hline Alabama & 6 & 0.5 \\
\hline Alaska & 1 & 0.1 \\
\hline Arizona & 31 & 2.5 \\
\hline Arkansas & 5 & 0.4 \\
\hline California & 29 & 2.3 \\
\hline Colorado & 12 & 1 \\
\hline Connecticut & 2 & 0.2 \\
\hline Delaware & 2 & 0.2 \\
\hline Florida & 24 & 1.9 \\
\hline Georgia & 27 & 2.2 \\
\hline Hawaii & 2 & 0.2 \\
\hline Idaho & 7 & 0.6 \\
\hline Illinois & 38 & 3.1 \\
\hline Indiana & 40 & 3.2 \\
\hline Iowa & 4 & 0.3 \\
\hline Kansas & 4 & 0.3 \\
\hline Kentucky & 8 & 0.6 \\
\hline Louisiana & 8 & 0.7 \\
\hline Maine & 3 & 0.3 \\
\hline Maryland & 5 & 0.4 \\
\hline Massachusetts & 13 & 1.1 \\
\hline Michigan & 12 & 0.9 \\
\hline Minnesota & 7 & 0.7 \\
\hline Mississippi & 1 & .1 \\
\hline Missouri & 18 & 1.4 \\
\hline Montana & 2 & 0.2 \\
\hline Nebraska & 3 & 0.2 \\
\hline Nevada & 2 & 0.2 \\
\hline New Hampshire & 1 & 0.1 \\
\hline New Jersey & 8 & 0.6 \\
\hline New York & 22 & 1.8 \\
\hline North Carolina & 9 & 0.7 \\
\hline Ohio & 19 & 1.5 \\
\hline Oklahoma & 11 & 0.9 \\
\hline Oregon & 5 & 0.4 \\
\hline Pennsylvania & 14 & 1.2 \\
\hline Rhode Island & 3 & 0.3 \\
\hline South Carolina & 9 & 0.7 \\
\hline South Dakota & 4 & 0.3 \\
\hline Tennessee & 11 & 0.9 \\
\hline Texas & 736 & 58.4 \\
\hline Utah & 10 & 0.8 \\
\hline Vermont & 2 & 0.2 \\
\hline Virginia & 17 & 1.3 \\
\hline Washington & 10 & 0.8 \\
\hline Washington, D.C. & 3 & .3 \\
\hline West Virginia & 5 & 0.4 \\
\hline Wisconsin & 10 & 0.8 \\
\hline Missing & 41 & 3.2 \\
\hline
\end{tabular}




\section{Analysis of Variance}

Women who identified as Democrat $(n=488$; mean $=35.92$; $\mathrm{SD}=3.30)$ and Independent $(\mathrm{n}=122$; mean 34.13 ; $\mathrm{SD}=5.63)$, or indicated not identifying with a political party $(n=279$; mean $=34.19 ; \mathrm{SD}=5.69)$, reported higher levels of family social distancing compared to women who identified as Republican $(\mathrm{n}=308 ;$ mean $=29.70 ; \mathrm{SD}=8.12 ; p$ 's $=.00)$. The largest effect was found between women who identified as Democrat and Republican (effect size $=1.00$ ). Women with a Doctorate $(\mathrm{n}=295$; mean $=35.37 ; \mathrm{SD}=4.24)$ and Master's $(n=396 ;$ mean $=34.26 ; \mathrm{SD}=5.70)$ degree reported higher levels of family social distancing compared to women with some college $(\mathrm{n}=87$; mean $=31.11 ; \mathrm{SD}=8.11)$ or a college degree $(\mathrm{n}=435 ;$ mean $=32.62 ; \mathrm{SD}=6.91 ; p$ 's $=.00)$. The largest effect was found between women who had obtained a Doctorate degree and women with some college (effect size $=.66$ ).

Women across the different annual income categories reported similar levels of family social distancing (Less than $\$ 50,000$ per year $\mathrm{n}=51$; mean $=32.88$; $\mathrm{SD}=7.90$; $\$ 50,000-\$ 74,999 \mathrm{n}=100 ;$ mean $=33.45 ; \mathrm{SD}=7.04$; $\$ 75,000-\$ 99,999 \mathrm{n}=164 ;$ mean $=33.96 ; \mathrm{SD}=6.17$; $\$ 100,000-\$ 150,000 \mathrm{n}=418$; mean $=33.74 ; \mathrm{SD}=6.16$; More than $\$ 150,000 \mathrm{n}=475$; mean $=33.74 ; \mathrm{SD}=5.91 ; p$ 's range from .82 to 1.00). Women across the different age categories reported similar levels of family social distancing (Ages 18-29 years $\mathrm{n}=30$; mean $=33.54$; $\mathrm{SD}=5.96$; Ages $30-39$ years $\mathrm{n}=588$; mean $=33.67 ; \mathrm{SD}=6.38$; Ages 40 years and older $\mathrm{n}=595$; mean $33.78 ; \mathrm{SD}=6.01 ; p$ 's range from .95 to .99 ).

\section{Multiple Linear Regression Analysis}

Results of the multiple linear regression analysis are presented in Table 4. The overall multiple linear regression

Table 4 Multiple linear regression analysis examining predictors of social distancing

\begin{tabular}{llc}
\hline Predictors & Social distancing & $p$ value \\
\hline Maternal education & .116 & .000 \\
Combined annual income & .014 & .626 \\
${ }^{\dagger}$ Political party identification & -.348 & .000 \\
Maternal age & .001 & .960 \\
${ }^{\dagger}$ Maternal race & .072 & .007 \\
Accounted variance & & \\
Cumulative (total model) $R^{2}$ & .160 & .000 \\
Cumulative (total model) $F$ & 46.21 & \\
\hline
\end{tabular}

'Dummy-coded variables with white and Republican as the reference groups (1) model was statistically significant $F(5,1187)=46.208$ $(p=.000)$. After controlling for all predictor variables, maternal education (standardized beta coefficient $=.116$; $p=.000$ ), race (standardized beta coefficient $=.072$; $p=.007$ ), and political party identification (standardized beta coefficient $=-.348 ; p=.000$ ) were significantly correlated with the Social Distancing Total Score. Specifically, higher maternal education and identifying as white and not Republican were associated with higher levels of family social distancing.

\section{Discussion}

The purpose of the current study was to assess maternal socio-demographic characteristics and political party identifications associated with family social distancing practices in a national sample of women. Our findings suggest that maternal race, educational status, and political party identification are important factors associated with family social distancing practices during the COVID-19 pandemic. The largest effects and differences in social distancing found in our study were a result of political party affiliation, with Republican women reporting the lowest levels of family social distancing. Many aspects of the response to COVID19 in the U.S., including local governments imposing stayat-home orders, have been politicized (Djulbegovic et al., 2020). Given that social distancing is a critical strategy for mitigating transmission of COVID-19 (CDC, 2020; Courtemanche et al., 2020), our findings suggest that it may be beneficial for public health campaigns that focus on social distancing to target families who identify as Republican or more conservative regions of the U.S. In particular, these campaigns may be most effective if they focus on the potential health benefits of social distancing practices while emphasizing the intent of these practices to not impinge on individual freedoms. Our findings, in conjunction with those of Gollwitzer et al. (2020) who found county-level consumption of conservative news media was associated with lower physical distancing, suggest political leadership around this topic from conservative leaders and media outlets has the potential to reduce partisanship around social distancing practices during the COVID-19 pandemic. Our findings suggest there may also be a benefit to social distancing public health campaigns targeting women with lower educational attainment. This is consistent with previous research that has reported a link between educational status and health behaviors such as eating healthy foods and having routine health check-ups (Lee \& Seon, 2019).

In examining mean social distancing scores in the current study, non-white women reported higher levels of social distancing for their families than white women. These differences did not hold after controlling for maternal education, 
combined annual income, political affiliation, and maternal age in the multiple linear regression analysis and identifying as white was associated with higher levels of family social distancing in women. There is mounting evidence that racial minority groups have higher rates of mortality from COVID19 (Price-Haywood et al., 2020). A recent national study among adults found that race was one of the strongest factors associated with COVID-19-related knowledge and behaviors, with Black adults demonstrating less knowledge compared to other groups (Alsan et al., 2020). Taken as a whole, our findings highlight the importance of not considering race in isolation when examining family social distancing practices as other factors including maternal age, education and income level may in part account for the association between race and family social distancing practices. Given the lack of racial diversity in the current study, all non-white women were grouped together to be able to make meaningful social distancing comparisons with white women. Future studies that recruit larger numbers of racial minority women are needed in order to determine if there are differences in family social distancing based on specific racial identities (e.g., Black, Latino).

Compared to women who reported someone in their home had been diagnosed with COVID-19, women in our sample who reported no one in their home had been diagnosed with COVID-19 reported higher levels of social distancing for their families. One interpretation of this finding is that women whose families practice more stringent social distancing practices are less likely to contract COVID-19. Another interpretation is that families who have a member who already had COVID-19 are less likely to practice social distancing since they may already have some level of immunity to COVID-19. While the directionality of this relationship is not able to be determined in the present study due to the cross-sectional study design, future longitudinal studies are needed to clarify the temporal associations between family social distancing practices and contracting COVID-19. Future studies should also consider how other practices such as contact tracing and testing in conjunction with social distancing practices influence COVID-19 rates among families (Matrajt \& Leung, 2020).

The current study had some limitations. Our sample was comprised predominantly of white, married women who had obtained high levels of education. For example, whereas approximately $13.1 \%$ of adults in the U.S. have obtained a Master's or Doctoral degree (United States Census Bureau, 2019a), a little over $50 \%$ of our sample of women had obtained a Master's or Doctoral degree. Approximately $76.3 \%$ of U.S. adults identify as white (United States Census Bureau, 2019b), while the percentage of white women in our sample was $85 \%$. The majority of women in our sample were also from Texas and our sampling strategy, which utilized social media, may have resulted in women participating in the study who had strong opinions on this topic. As such, our findings may not be generalizable to U.S. mothers as a whole, including more diverse samples of women and those who reside outside of Texas. We did not collect specific country of origin or social entities information on participants in our sample, including Latino participants, and as a result were not able to report this information. Although our social distancing measure was based on the Centers for Disease Control and Prevention (CDC) COVID-19 Tips for Social Distancing and demonstrated strong internal consistency reliability in the current study, it has not been validated in previous research. Further, while the social distancing measure referred to practices by 'my family and I', levels of social distancing may have varied among individual family members in the household, and our study did not examine within-household variation of social distancing practices. Another limitation of our study was the inability to objectively confirm participants met all of the study inclusion criteria.

In conclusion, we found that maternal race, educational status, and political party identification are important factors associated with family social distancing practices during the COVID-19 pandemic. Women with a Doctorate and Master's degree reported higher levels of family social distancing compared to women with some college or a college degree. Women who identified as Democrat or Independent, or indicated not identifying with a political party, reported higher levels of family social distancing compared to women who identified as Republican. The largest effect was found between women who identified as Democrat and Republican. After controlling for maternal education, combined annual income, political affiliation, and maternal age in the multiple linear regression analysis, identifying as white was associated with higher levels of family social distancing in women.

Funding No funding was received for the present study.

\section{Declarations}

Conflict of interest The authors declare that they have no conflict of interest.

\section{References}

Alsan, M., Stantcheva, S., Yang, D., \& Cutler, D. (2020). Disparities in Coronavirus 2019 reported incidence, knowledge, and behavior among US adults. JAMA Network Open, 3, e2012403.

Centers for Disease Control and Prevention. (2020). Social distancing: Keep a safe distance to slow the spread. Centers for Disease Control and Prevention. 
Chu, D. K., Akl, E. A., Duda, S., Solo, K., Yaacoub, S., \& Schünemann, H. J. (2020). Physical distancing, face masks, and eye protection to prevent person-to-person transmission of SARSCoV-2 and COVID-19: A systematic review and meta-analysis. The Lancet, 395(10242), 1973-1987. https://doi.org/10.1016/ s0140-6736(20)31142-9

Clinton, J., Cohen, J., Lapinski, J., \& Trussler, M. (2020). Partisan pandemic: How partisanship and public health concerns affect individuals' social distancing during COVID-19. Available at SSRN: https://ssrn.com/abstract $=3633934$

Courtemanche, C., Garuccio, J., Le, A., Pinkston, J., \& Yelowitz, A. (2020). Strong social distancing measures in the United States reduced the COVID-19 growth rate. Health Affairs, 39(7), 12371246. https://doi.org/10.1377/hlthaff.2020.00608

Djulbegovic, B., Weiss, D., \& Hozo, I. (2020). Evaluation of the U.S. governors' decision when to issue stay-at-home orders. Journal of Evaluation in Clinical Practice, Advanced Online Publication, 26(5), 1347-1351.

Gollwitzer, A., Martel, C., Brady, W., Parnamets, P., Freedman, I., Knowles, E., \& Bavel, J. (2020). Partisan differences in physical distancing are linked to health outcomes during the COVID-19 pandemic. Nature Human Behavior, 4, 1186-1197.

Lee, J., \& Seon, J. (2019). Educational attainment and health behaviors among young adult men: Racial/Ethnic disparities. American Journal of Men's Health, 13, 1557988319894488.

Leventhal, A., Dai, H., Barrington-Trimis, J., McConnell, R., Unger, J., Sussman, S., \& Cho, J. (2021). Association of political party affiliation with physical distancing among young adults during the COVID-19 pandemic. JAMA Internal Medicine, 181, 399-403.
Matrajt, L., \& Leung, T. (2020). Evaluating the effectiveness of social distancing interventions to delay or flatten the epidemic curve of coronavirus disease. Emerging Infectious Diseases, 26, $1740-1748$.

Price-Haywood, E., Burton, J., Fort, D., \& Seoane, L. (2020). Hospitalization and mortality among black patients and white patients with Covid-19. New England Journal of Medicine, 382, 2534-2543.

Rashid, H., Ridda, I., King, C., Begun, M., Tekin, H., Wood, J. G., \& Booy, R. (2015). Evidence compendium and advice on social distancing and other related measures for response to an influenza pandemic. Paediatric Respiratory Reviews, 16(2), 119-126. https://doi.org/10.1016/j.prrv.2014.01.003

United States Census Bureau. (2019a). Number of people with master's and doctoral degrees doubles since 2000. Retrieved June 30, 2021, from, https://www.census.gov/library/stories/2019/02/ number-of-people-with-masters-and-phd-degrees-double-since2000.html

United States Census Bureau. (2019b). Quick facts. Retrieved June 30, 2021, from, https://www.census.gov/quickfacts/fact/table/US/ RHI125219\#RHI125219

Publisher's Note Springer Nature remains neutral with regard to jurisdictional claims in published maps and institutional affiliations. 